\title{
Reflets
}

Revue ontaroise d'intervention sociale et communautaire

\section{Résultats du sondage Reflets}

\section{Claude Camirand}

Volume 3, numéro 1, printemps 1997

Enfance et familles en contexte d'appauvrissement

URI : https://id.erudit.org/iderudit/026164ar

DOI : https://doi.org/10.7202/026164ar

Aller au sommaire du numéro

Éditeur(s)

Reflets : Revue ontaroise d'intervention sociale et communautaire

ISSN

1203-4576 (imprimé)

1712-8498 (numérique)

Découvrir la revue

Citer cet article

Camirand, C. (1997). Résultats du sondage Reflets. Reflets, 3(1), 168-173.

https://doi.org/10.7202/026164ar

Tous droits réservés (C) Reflets : Revue ontaroise d'intervention sociale et communautaire, 1997

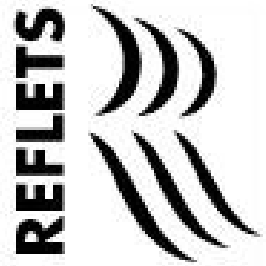

Ce document est protégé par la loi sur le droit d'auteur. L'utilisation des services d'Érudit (y compris la reproduction) est assujettie à sa politique d'utilisation que vous pouvez consulter en ligne.

https://apropos.erudit.org/fr/usagers/politique-dutilisation/ 


\title{
Résultats du sondage Reflets
}

\author{
Claude Camirand \\ École de service social, Ottawa
}

Entre le 31 octobre et le 7 novembre 1996, Reflets a mené un sondage téléphonique auprès de ses abonnées et abonnés afin d'obtenir un feedback sur la revue et de cueillir de l'information sur son contenu et son utilité. Les renseignements obtenus serviraient aussi à faciliter la réflexion du Comité de direction quant à l'atteinte des objectifs fixés lors de la création de Reflets. Les questions posées visent à obtenir les impressions, suggestions et préférences des lectrices et lecteurs de Reflets.

\section{Échantillon}

Une cinquantaine d'abonnés fut choisie au hasard, avec l'emplacement géographique des individus comme critère principal de sélection. L'objectif était de sélectionner des répondantes et des répondants de chaque région de l'Ontario. Parmi les 22 personnes que nous avons réussi à rejoindre par voie téléphonique, toutes ont accepté volontiers de participer au sondage.

La distribution géographique de l'échantillon comprenait une personne provenant de chacune des régions suivantes : Cornwall, Chatham, Gloucester, Hawkesbury, Hearst, Iroquois Falls, Kapuskasing, Ottawa, Sturgeon Falls, Wawa; deux personnes provenant de la région de Thunder Bay et trois personnes provenant de chacune des régions de Sudbury, Timmins et Toronto. 


\section{Résumé des réponses au questionnaire}

Pourriez-vous me donner vos impressions générales sur la revue Reflets? La majorité des individus ont répondu à cette question de façon très positive. Pour eux, Reflets est une revue qui touche à peu près à tout et qui offre une meilleure perspective de l'Ontario. Presque toutes les personnes contactées ont mentionné qu'elles trouvaient très intéressant de connaître les pratiques des autres régions, afin de "savoir ce qui se passe ailleurs dans la province».

En effet, plusieurs répondantes ont souligné que les FrancoOntariennes et Franco-Ontariens représentent une population éparpillée sur un vaste territoire, qu'il existe peu de liens entre les francophones des différentes régions et qu'ils sont difficiles à rejoindre, surtout en ce qui a trait aux populations francophones des régions rurales nordiques, plutôt isolées. Ces derniers ont relativement peu de contacts avec les grands centres. En ce sens, certains répondants ont indiqué que Reflets leur permettait d'avoir un certain contact avec les autres francophones de la province et de faire du réseautage.

- «La revue permet aux agences de collaborer dans la rédaction d'articles et de faire des liens avec ce qui se passe ailleurs.»

- «Les articles ciblent bien la réalité du Nord de l'Ontario et nous informent des approches dans les milieux ruraux.»

De plus, puisque la littérature francophone n'est pas des plus abondantes en Ontario, bon nombre de répondantes et répondants ont soulevé l'importance n'est de non seulement pouvoir finalement lire en français, mais aussi de pouvoir lire des articles par des gens de chez-nous. Une des répondantes a ajouté que Reflets «remplit un vide au niveau de la recherche et de l'intervention francophone en Ontario».

Y a-t-il une rubrique qui vous intéresse plus particulièrement?

Les réponses à cette deuxième question ont été très révélatrices. Bien que plusieurs des individus questionnés aient rapporté qu'ils lisaient la revue «d'un bout à l'autre» sans discrimination, la 
rubrique Aux quatres coins de la province s'est avérée de loin la plus populaire, suivie du Dossier et Des pratiques à notre image.

En tenant compte des commentaires reçus pour la première question, soit les impressions générales, il n'est pas surprenant de constater que la rubrique Aux quatres coins de la province soit la plus populaire auprès des abonnés. En effet, cette rubrique répond directement au désir des lectrices et lecteurs de connaître les pratiques d'intervention dans les autres régions de la province.

De quelles façons avez-vous été en mesure d'utiliser le contenu de Reflets?

La plupart des répondantes et répondants ont mentionné qu'ils lisaient Reflets pour des fins personnelles et pour connaître les pratiques des intervenantes et intervenants des autres régions de l'Ontario. Comme mentionné ci-haut, l'utilité de Reflets s'est aussi manifestée au niveau du réseautage. Les gens sollicités lors du sondage ont rapporté qu'en plus de permettre aux gens de connaitre les services offerts et les pratiques dans les autres régions, la Revue permettait de connaître qui faisait partie de quel organisme et avec qui il était possible de collaborer dans l'organisation de colloques et de recherches.

- «Suite à l'entrevue avec Lynne Toupin, nous avons invité l'Organisation nationale anti-pauvreté à une assemblée générale.»

- «La revue nous a permis d'entrer en contact avec diverses personnes et de tenir un forum.»

- «Nous nous sommes inspirés d'une idée parue dans la revue sur l'organisation communautaire des centres de services en français.»

- «Un des articles nous a permis de voir qu'une autre personne faisait de la recherche dans le même domaine que nous et nous sommes entrés en communication avec elle.»

Bon nombre de répondantes et répondants ont indiqué qu'ils trouvaient Reflets utile pour préparer des documents et pour faire de la recherche. Certains ont aussi ajouté qu'ils allaient puiser des informations dans Reflets pour donner des lectures à leurs étudiantes et étudiants. D'autres ont distribué des copies de Reflets 
dans la communauté pour sensibiliser les femmes francophones à la violence. D'autres, encore, choisissent de faire lire certains articles aux nouveaux employés pour qu'ils puissent se familiariser avec diverses problématiques sociales.

Quels thèmes ou dossiers aimeriez-vous voir paraître dans Reflets?

Les suggestions pour les thèmes et dossiers furent très variés et presque tous les répondants et répondantes ont soumis des idées.

Tout d'abord, il y a eu de nombreuses suggestions à l'égard des femmes et des familles. Quelques répondantes ont fait remarquer qu'il serait très intéressant de souligner les réalisations de la femme à travers les âges, c'est-à-dire, décrire ses nombreux aspects positifs plutôt que de l'exposer comme victime. Dans cet optique, les répondantes ont ajouté qu'il serait très intéressant d'avoir un dossier qui accentue ses réalisations et qui souligne son rôle dans la société; son évolution dans divers domaines tels que l'éducation, l'économie, la politique, la famille, etc...

La réforme gouvernementale et le budget représentent un autre aspect fréquemment suggéré comme thème possible du dossier. Les répondantes et répondants du sondage Reflets aimeraient voir paraitre un dossier portant sur les répercussions des coupures budgétaires provinciales et de la restructuration des services sociaux et de santé sur la population franco-ontarienne. Plusieurs sont préoccupés par les impacts des décisions gouvernementales sur la violence et l'emploi chez les francophones de l'Ontario, surtout d'un point de vue rural et nordique.

D'autres ont souligné qu'il serait intéressant de traiter des réformes politiques au niveau des divers acteurs du système, c'està-dire les mouvements francophones dans nos communautés qui mettent des pressions sur les gouvernements. Un dossier de ce genre permettrait de connaitre les actions des Franco-Ontariennes et des Franco-Ontariens au niveau politique, de savoir comment les autres organismes de la province réussissent à survivre malgré les coupures, les budgets restreints, la restructuration et les compressions. 
Parmi les autres thèmes suggérés, mentionnons l'emploi (pour jeunes et adultes) et les problèmes sociaux qui s'y rattachent, les difficultés et les défis de la recherche auprès des francophones en Ontario, les ressources alternatives en Ontario, les personnes handicapées francophones, l'alphabétisation pour les francophones en Ontario, la problématique du VIH/Sida, les personnes sansabri, l'économie sociale, ainsi que l'adolescence et la santé (cigarette, toxicomanie, alcool, etc...).

Il est à noter que certaines des suggestions ont en effet déjà été choisies comme thème des dossiers à venir. Par exemple, le volume 4 numéro 2 aura comme thème Personnes vivant avec une incapacité et le numéro suivant sera intitulé Intervention et économie.

Auriez-vous des suggestions pour améliorer le contenu de Reflets?

De façon générale, les répondantes et répondants se disaient très satisfaits du contenu de la revue, et les suggestions pour l'améliorer n'étaient pas des plus abondantes. La plupart des personnes contactées lors du sondage n'avaient pas de suggestions.

Néanmoins, parmi les quelques informations recueillies, certains ont exprimé qu'ils aimeraient voir paraître plus de témoignages et d'entrevues dans les numéros de Reflets. Il a aussi été suggéré de présenter des articles un peu moins littéraires et plus accessibles au niveau du langage, d'avoir moins d'articles théoriques et plus de matériel qui se rapporte directement à l'intervention et à la vie de tous les jours.

«Les articles sont très intéressants mais parfois trop scientifiques pour ce que nous faisons dans nos pratiques.» Par contre, les opinions à ce niveau étaient variées puisque d'autres ont manifesté un intérêt plus particulier pour des articles plus académiques ou théoriques.Toutefois, plusieurs ont affirmé que le mélange théorie/ pratique était bien équilibré. 


\section{Conclusion}

Les informations recueillies lors de ce sondage ont suscité une réflexion au sein du Comité de direction lui permettant d'évaluer son parcours en ce qui a trait à l'atteinte de ses objectifs. Cet exercice a aussi permis de connaitre certaines des préférences des lectrices et des lecteurs de la Revue ontaroise d'intervention sociale et communautaire.

Peu importe votre domaine de pratique, qu'il s'agisse de l'intervention communautaire ou institutionnelle, de la recherche ou de l'enseignement, vous êtes invités à soumettre vos commentaires de façon régulière, à émettre des suggestions pour des thèmes particuliers et à soumettre des articles. Les francophones de toutes les régions de l'Ontario ont envie de connaître vos pratiques d'intervention, de savoir ce qui se passe aux quatre coins de la province et d'établir des contacts avec divers intervenantes et intervenants de toutes les régions.

En terminant, je remercie tous ceux et celles qui, malgré les horaires surchargés et les nombreux engagements, ont pris le temps de participer à ce sondage. 